\title{
Comparison of a Silver-Coated Needleless Connector and a Standard Needleless Connector for the Prevention of Central Line-Associated Bloodstream Infections
}

\author{
Jesse T. Jacob, MD; ${ }^{1,5}$ Sheri Chernetsky Tejedor, MD; $;{ }^{2,3}$ Mary Dent Reyes, MPH; ${ }^{1}$ Xin Lu, MS $;{ }^{4}$ Kirk A. Easley, MS; ${ }^{4}$ \\ William L. Aurand, MPH; ${ }^{5}$ Gina Garrett, RN; $;^{3}$ Kimberly Graham, RN; ${ }^{3}$ Carolyn Holder, RN; ${ }^{3}$ Chad Robichaux, MPH; ${ }^{3}$ \\ James P. Steinberg, $\mathrm{MD}^{1}$
}

овјестіvе. To assess the impact of a novel, silver-coated needleless connectors (NCs) on central-line-associated bloodstream infection (CLABSI) rates compared with a mechanically identical NCs without a silver coating.

DESIGN. Prospective longitudinal observation study

SETTING. Two 500-bed university hospitals

PAtients. All hospitalized adults from November 2009 to June 2011 with non-hemodialysis central lines

interventions. Hospital A started with silver-coated NCs and switched to standard NCs in September 2010; hospital B started with standard NCs and switched to silver-coated NCs. The primary outcome was the difference revealed by Poisson multivariate regression in CLABSI rate using standard Centers for Disease Control and Prevention surveillance definitions. The secondary outcome was a comparison of organism-specific CLABSI rates by NC type.

RESUlts. Among 15,845 hospital admissions, 140,186 central-line days and 221 CLABSIs were recorded during the study period. In a multivariate model, the CLABSI rate per 1,000 central-line days was lower with silver-coated NCs than with standard NCs (1.21 vs 1.79 ; incidence rate ratio $=0.68$ [95\% CI: 0.52-0.89], $P=.005$ ). A lower CLABSI rate per 1,000 central-line days for the silver-coated NCs versus the standard NCs was observed with $S$. aureus $(0.11$ vs $0.30, P=.02)$, enterococci $(0.10$ vs $0.27, P=.03)$, and Gram-negative organisms ( 0.28 vs $0.63, P=.003)$ but not with coagulase-negative staphylococci ( 0.31 vs 0.36$)$ or Candida spp. (0.42 vs 0.40$)$.

CONCLUSIONS. The use of silver-coated NCs decreased the CLABSI rate by $32 \%$. CLABSI reduction efforts should include efforts to minimize contamination of NCs.

Infect Control Hosp Epidemiol 2015;36(3):294-301

Central-line-associated bloodstream infection (CLABSI) is a major cause of patient harm, and prevention of such infections has received much attention over the past decade. A national effort to prevent CLABSIs has been undertaken through optimization of central-line insertion practices. With this and other strategies, including economic disincentives through payment reform and public reporting of infection rates, the incidence of CLABSI has decreased in U.S. hospitals; however, in 2009, 41,000 CLABSIs were reported. ${ }^{1}$ The persistence of CLABSIs and their high associated mortality underscore the need for novel prevention efforts.

CLABSIs occur through two major routes. Infection can occur via an extraluminal pathway when microorganisms, often from the skin surface, gain access to the bloodstream via the external catheter surface. This contamination of the catheter surface can occur at the time of catheter insertion, particularly if proper insertion practices are not used. Intraluminal colonization of the catheter with seeding of the bloodstream typically occurs following contamination of the catheter hub, which is frequently manipulated in the process of injecting medications or fluids. Molecular typing of blood, skin, and catheter hub isolates suggests that most CLABSIs occurring early after line insertion originate from the skin (and are likely insertion-related events), whereas many CLABSIs occurring after the first 1-2 weeks of line placement are related to catheter hub contamination. ${ }^{2-8}$

Affiliations: 1. Division of Infectious Diseases, Department of Medicine, Emory University School of Medicine, Atlanta, Georgia; 2. Division of Hospital Medicine, Department of Medicine, Emory University School of Medicine, Atlanta, Georgia; 3. Emory Healthcare, Atlanta, Georgia; 4. Department of Biostatistics and Bioinformatics Emory University School of Public Health, Atlanta, Georgia; 5. Department of Epidemiology, Emory University School of Public Health, Atlanta, Georgia.

Received August 20, 2014; accepted November 20, 2014; electronically published December 30, 2014

(c) 2014 by The Society for Healthcare Epidemiology of America. All rights reserved. 0899-823X/2015/3603-0009. DOI: 10.1017/ice.2014.58 
Most notable CLABSI prevention efforts to date have focused on optimizing insertion practices. ${ }^{9}$ While these efforts have successfully decreased CLABSI rates, they have not eliminated preventable CLABSIs. One study demonstrated that, after specific preventive measures were used to address contamination at the insertion site, $60 \%$ of CLABSIs were more likely to originate from the intraluminal pathway. ${ }^{7}$ Consequently, preventing intraluminal contamination of catheters through proper catheter maintenance or improved product design is a priority because much of the research and prevention efforts have been focused on extraluminal contamination at the time of catheter insertion.

Intravenous catheters use needleless connectors (NCs) to allow access to the fluid pathway. Cleaning the external surface of the NC prior to accession of the fluid pathway is standard practice to minimize device contamination. ${ }^{10}$ However, device cleaning may be done inconsistently, and some devices, through suboptimal design, may be more prone to contamination. Some NCs, particularly positive-pressure valve mechanism devices, have been associated with an increased risk of CLABSI, presumably by predisposing the device to colonization. ${ }^{11-14}$ Novel NCs designed to reduce microbial colonization are attractive candidates to help prevent intraluminal contamination and bloodstream infection.

Although its exact antimicrobial mechanism is unknown, silver has been used topically as an antimicrobial for decades and has been incorporated into urinary catheters. ${ }^{15-17}$ The use of chlorhexidine-silver-sulfadiazine-coated central lines has previously been shown to reduce CLABSI rates. ${ }^{18}$ An antimicrobial Luer-activated NC with a novel nanoparticle silver coating of the internal fluid pathway (Vlink with VitalShield, Baxter Healthcare) has been shown to decrease bacterial growth and biofilm formation in vitro. ${ }^{19,20}$ This silver-coated NC has been approved by the Food and Drug Administration, but the efficacy of this device in reducing the CLABSI rate has not been shown in a clinical setting. We sought to assess the impact of this silver-coated NC on CLABSI rates by comparing it to a mechanically identical NC (Clearlink, Baxter Healthcare) without a silver coating.

\section{METHODS}

The study was a non-randomized, prospective, controlled cohort study of 2 NCs between 2 hospitals. Previously, the 2 hospitals used a standard Luer-activated, negative-pressure NC (Clearlink, Baxter Healthcare). At the start of the study, hospital A switched to the silver-coated NC for all non-dialysis intravenous catheters and hospital B continued using the standard NC. At a predetermined number of CLABSIs based on sample size calculation, hospital A switched to the standard NC and hospital B switched to the silver-coated NC. This switch occurred in September 2010.

All adults admitted to two $~ 500$-bed university hospitals between November 1, 2009, and June 30, 2011, were enrolled in the study. Hospital B has a large transplant and oncology population. Both hospitals used chlorhexidine-silver sulfadiazine-impregnated, triple-lumen catheters and uncoated peripherally inserted central catheters and emphasized compliance with the central-line insertion care bundle before and during the study. There were no major hospital-wide process improvement activities targeting CLABSI reduction in either hospital during the study period that would potentially confound the results.

In the first week of each transition month (November 2009 and September 2010), nurses were provided in-service education regarding the NCs and the study, and materials management replaced all supplies of the previous $\mathrm{NC}$ with the subsequent $\mathrm{NC}$ in both hospitals. The study coordinator performed periodic audits during the transition periods to look for caches of the previous NC. A full transition month, during which data were not collected, was used to deplete unrecognized supplies. The pre-existing nursing protocol was continued through the study period; it required NC change every 7 days or more frequently if the blood persisted in the NC after flushing or if the NC was removed to access the catheter directly.

The primary outcome was the CLABSI rate comparing the silver-coated NC with the standard NC. The secondary outcome was the comparison of organism-specific CLABSI rates between the silver-coated NC and the standard NC. CLABSIs were defined using the contemporary surveillance definitions from the Centers for Disease Control and Prevention's (CDC's) National Healthcare Safety Network (NSHN). ${ }^{21}$ The determinations of all CLABSIs during the study were made by a single hospital epidemiologist (JPS) blinded to institution. Central-line days were captured electronically using a previously validated process. ${ }^{22}$ Discrepancies in electronic line-day documentation were flagged by automated tools, manually reviewed by a member of the study team (MDR), and reconciled.

CLABSI rates were calculated by dividing the raw number of CLABSIs by the number of central-line days during the same period and multiplying the result by 1,000 . Because of the lack of specificity of the NHSN CLABSI definition in patients with neutropenia induced by chemotherapy and the anticipated higher frequency of these patients in hospital $\mathrm{B},{ }^{23-26}$ a prespecified sensitivity analysis was performed excluding patients with CLABSIs occurring in the setting of chemotherapyinduced neutropenia from the numerator. The NHSN definition (with a recently proposed amendment) attributes as CLABSIs those bloodstream infections that could be due to translocation of intestinal microbiota in the setting of chemotherapy-induced mucositis. ${ }^{27}$ For our study, neutropenia was defined as an absolute neutrophil count of $\leq 500$ cells $/ \mathrm{mm}^{3}$ on the day the blood cultures were obtained in patients who had received cytotoxic chemotherapy. Centralline days during periods of neutropenia were neither measured nor excluded from the denominator.

Because neither NC was compatible with hemodialysis catheters, infections, and central-line days attributed to these catheters were censored from both the numerator (CLABSIs) 
and denominator (central-line days) of the CLABSI rate. CLABSIs that occurred in patients with both hemodialysis catheters and non-hemodialysis catheters were also excluded. Etiologic organism(s) and catheter type for each CLABSI was also recorded, and these data were stratified by NC type. For CLABSIs attributed to a single catheter inserted at admission or during the hospitalization, the time from catheter insertion to infection was determined and stratified by organism type.

To assess whether the impact of the silver-coated NC on some but not all pathogens could be explained by time of occurrence after line insertion, we stratified the time from central-line insertion to CLABSI by pathogen. We hypothesized that, if coagulase-negative staphylococcal CLABSIs occurred predominantly in the first 7-10 days after line insertion due to insertion-related events (eg, skin colonization), their prevention would not be amenable to an antimicrobial NC.

\section{Statistical Analysis}

CLABSI rates per 1,000 central-line days and the distribution of CLABSI rates by organism were estimated and compared using exact methods based on the Poisson distribution. ${ }^{28}$ The incidence rates were compared between subgroups, using methods described by Brownlee. ${ }^{29}$ Specifically, an exact (significance) test was used to compare the equality of 2 Poisson rates by testing whether the ratio of 2 Poisson rates was different from 1. Covariates included sex, race, age, type of catheter, hospital, and intensive care unit (ICU) admission. The interaction between type of catheter and hospital was tested to determine whether the effect of catheter type on CLABSI rate differed between the 2 hospitals. SAS ${ }^{\circledast} 9.2$ (Cary, NC) was used for all analyses.

Incidence rates of infection by type of device were estimated and compared by performing a generalized estimating equation with Poisson regression analysis of the counts, using an exchangeable correlation structure for the repeated counts in SAS Proc Genmod. The incidence rate ratio (IRR) is the ratio of the incidence density in 1 group to that of another group. Results by each covariate are presented as the IRR and the $95 \%$ confidence interval $(\mathrm{CI})$. Covariates with $P<.05$ in the univariate analyses were included in multivariate analyses after checking for interaction. The IRR and the $95 \%$ CI were calculated for each factor, adjusting for other factors in the final model.

Sample size and power calculations for the study follow the method described by Rosner ${ }^{30}$ for comparing 2 incidence rates (silver and standard NCs) using an IRR. The estimated incidence rate for the standard NC based on data collected at the 2 hospitals between January 2008 and June 2008 was approximately 0.7 per 1,000 CLABSIs per 1,000 patient days (88 infections, 133,426 patient days, and 18,716 admissions). Based on the preliminary data using the standard NC, to detect a $50 \%$ reduction in the silver-coated NC CLABSI rate relative to the standard NC CLABSI rate, the required total number of CLABSIs was 131 (44 CLABSIs in the silver-coated NC group and 87 CLABSIs in the standard NC group) to achieve $80 \%$ power (2-sided test with significance level of 0.05 ) to detect an IRR of 0.50 .

\section{Human Subject Protection}

This study was approved by Emory University's Institutional Review Board with a waiver of individual patient consent.

\section{RES ULT S}

A total of 15,845 unique hospital admissions in which patients had $\geq 1$ central line were included in the study, with a total of 140,186 central-line days. Hospital B accounted for the 92,906 central-line days $(66.3 \%)$. The distribution of central-line days, total hospital days, ICU patient days, and number of admissions were similar in both study groups (Table 1).

A total of 221 CLABSIs occurred during the study period, including 66 in neutropenic patients, and 28 CLABSIs were attributed to $>1$ organism. A total of 106 CLABSIs occurred in patients with only 1 central line that was inserted either at time of admission or during the course of the hospitalization. Among all CLABSIs, 90 (40.7\%) occurred over 70,737 centralline days in patients with whom the silver-coated NC was used, and 131 (59.3\%) occurred over 69,449 central-line days in patients with whom the standard NC was used. Of 157 CLABSIs in patients without neutropenia (64.2\%), 62 of 64 of CLABSIs in neutropenic patients (96.9\%) occurred in hospital B. In univariate analysis (Table 2), the CLABSI rate per 1,000 central-line days was higher for the standard NC group (1.89) than for the silver-coated NC group (1.27, $P=.005)$. No differences in CLABSI rates associated with hospital, race, or ICU admission were observed. Similar results were found in the sensitivity analysis excluding patients with CLABSI and neutropenic fever, except that the CLABSI rate per 1,000 central-line days was higher for patients admitted to the ICU (1.47) than for those not admitted to the $\operatorname{ICU}(0.64, P<.001)$.

The multivariate model included NC and hospital; no statistical evidence of interaction between these 2 variables was detected $(P=.91)$. The adjusted CLABSI rate per 1,000 central-line days was lower for the silver-coated NC group than for the standard NC group (1.21 vs 1.79), with a corresponding IRR of 0.68 (95\% CI: $0.52-0.89, P=.005)$. The differences in rates between hospital A and B did not achieve statistical significance. Results were similar for the sensitivity analysis, except that the adjusted CLABSI rate was higher in hospital A than in hospital B (Table 3).

The microbiology of CLABSIs differed somewhat for the silver-coated NC compared with the standard NC (Table 4). Patients in whom the silver-coated NC was used had significantly fewer CLABSIs per 1,000 central-line days than those in whom the standard NC was used for $S$. aureus ( 0.11 vs $0.30, P=.02$ ), 
ta ble 1. Description of Patient Characteristics Comparing Those Receiving Silver and Standard Needleless Connectors

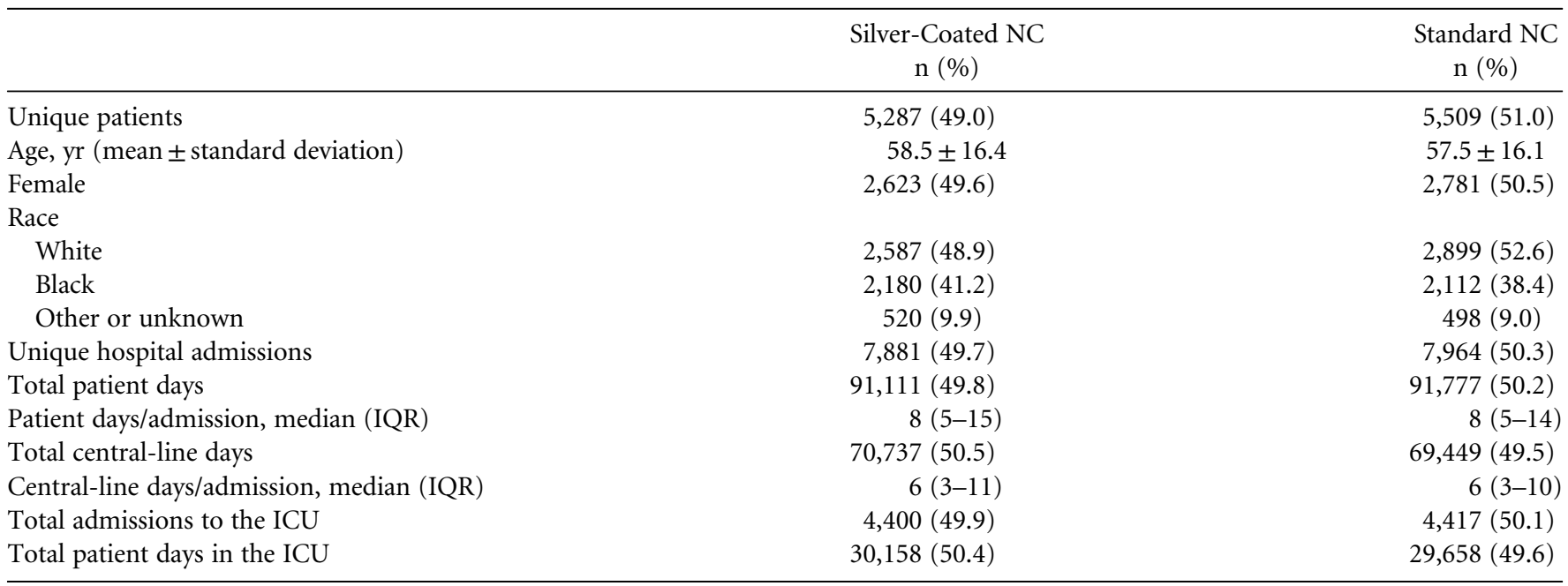

NOTE. ICU, intensive care unit; IQR, interquartile range; NC, needleless connectors.

TAвLE 2. Univariate analysis of risk factors for central line-associated bloodstream infection rates (CLABSI) per 1,000 central-line days.

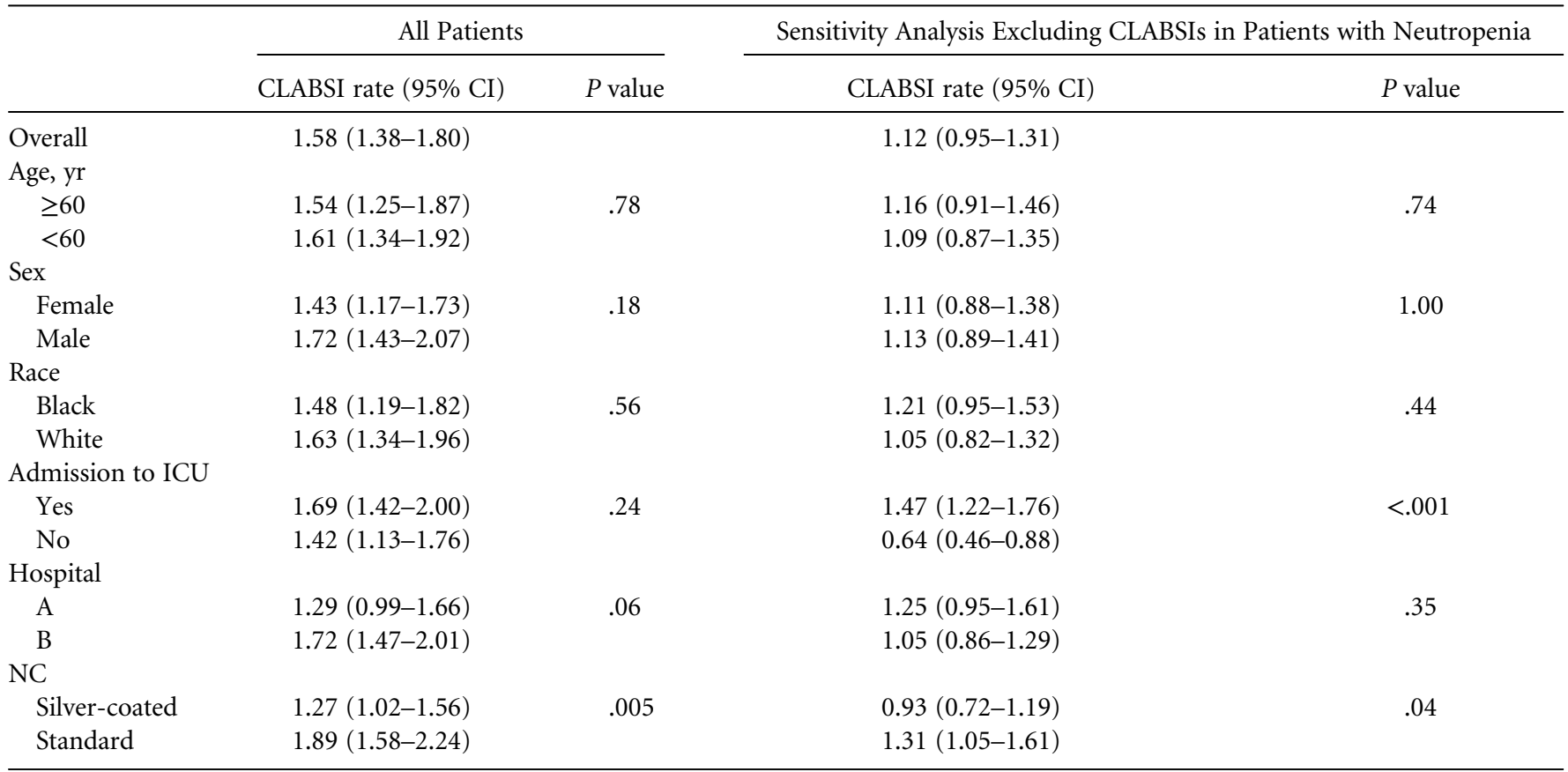

NOTE. CI, confidence interval; ICU, intensive care unit; NC, needleless connector.

TABLE 3. Multivariate Analysis of Factors Associated with Central-Line-Associated Bloodstream Infection (CLABSI) Rates Per 1,000 Central-Line Days and Their Incidence Rate Ratios (IRR)

\begin{tabular}{|c|c|c|c|c|c|c|}
\hline & \multicolumn{3}{|c|}{ All Patients } & \multicolumn{3}{|c|}{ Sensitivity Analysis Excluding CLABSIs in Patients with Neutropenia } \\
\hline Silver & $1.21(0.97-1.51)$ & $0.68(0.52-0.89)$ & .005 & $0.96(0.75-1.23)$ & $0.71(0.51-0.98)$ & .04 \\
\hline Standard & $1.79(1.48-2.15)$ & Reference & & $1.35(1.09-1.69)$ & Reference & \\
\hline \multicolumn{7}{|l|}{ Hospital } \\
\hline
\end{tabular}

NOTE. CI, confidence interval; NC, needleless connector. 
TAвLE 4. Distribution of Microbiology of Central-Line-Associated Bloodstream Infection Comparing Patients with the Silver-Coated Needleless Connector (NC) Compared with the Standard NC.

\begin{tabular}{|c|c|c|c|}
\hline & Silver-coated NC $\left(\mathrm{n}=90^{\mathrm{a}}\right)$ & Standard NC $\left(\mathrm{n}=131^{\mathrm{a}}\right)$ & Both NCs $\left(n=221^{a}\right)$ \\
\hline Gram-positive organisms & $47(52.2)$ & $67(51.1)$ & $114(51.6)$ \\
\hline Methicillin-resistant & $7(7.8)$ & $15(11.5)$ & $22(10.0)$ \\
\hline Methicillin-sensitive & $1(1.1)$ & $6(4.6)$ & $7(3.2)$ \\
\hline Streptococci, alpha hemolytic & $4(4.4)$ & $2(1.5)$ & $6(2.7)$ \\
\hline Other & $8(8.9)$ & $5(3.8)$ & $13(5.9)$ \\
\hline Gram-negative organisms & $20(22.2)$ & $44(33.6)$ & $64(29.0)$ \\
\hline Enterobacteriaceae & $17(18.9)$ & $41(31.3)$ & $58(26.2)$ \\
\hline E. coli & $5(5.6)$ & $15(11.5)$ & $20(9.0)$ \\
\hline Klebsiella spp. & $7(7.8)$ & $10(7.6)$ & $17(7.7)$ \\
\hline Yeast & $30(33.3)$ & $28(21.4)$ & $58(26.2)$ \\
\hline C. albicans & $14(15.6)$ & $12(9.2)$ & $26(11.8)$ \\
\hline C. glabrata & $10(11.1)$ & $6(4.6)$ & $16(7.2)$ \\
\hline C. parapsilosis & $3(3.3)$ & $5(3.8)$ & $8(3.6)$ \\
\hline Other & $3(3.3)$ & $8(6.1)$ & $11(5.0)$ \\
\hline
\end{tabular}

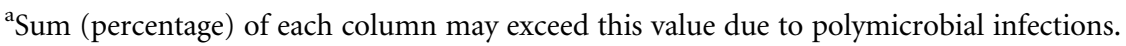

enterococci ( 0.10 vs $0.27, P=.03)$, and Gram-negative organisms $(0.28$ vs $0.63, P=.003)$, but not for coagulase-negative staphylococci $(0.31$ vs $0.36, P=.72)$, other non-staphylococcal Gram-positive organisms ( 0.17 vs $0.10, P=.38$ ), or Candida spp. (0.42 vs $0.40, P=.95$ ) (Figure 1$)$. No clustering of coagulase-negative staphylococci or other pathogen was observed within the first week after insertion.

\section{I S C U S S I O N}

Our results show a $32 \%$ reduction in CLABSI with the use of a silver-coated NC compared with a standard, mechanically identical NC. This is the first published study using clinical outcomes to assess the efficacy of silver-coated NCs. In addition, this study was large: it included $>10,000$ patients with $>140,000$ central-line days, and it was conducted at 2 academic medical centers with relatively low baseline CLABSI rates compared with those in prior published literature. ${ }^{9,31}$

Previously, a lower burden of organisms has been found in effluent from silver-coated NCs. ${ }^{32}$ From a convenience sample of 62 patients enrolled in the current study, $151 \mathrm{NCs}$ were prospectively obtained for biofilm analysis; biofilm was observed on the luminal surface of $>90 \%$ of both silvercoated and standard NCs. ${ }^{33}$ Microorganisms were recovered by culture from $\sim 50 \%$ of both NC types, substantially less than those with biofilm burden, but none of the patients from whom these NC cultures were obtained developed a CLABSI. Thus, the relationship between biofilm formation within a NC and subsequent bloodstream infection remains unclear. While the silver coating of the NC does not appear to reduce biofilm formation, the reduction in bloodstream infections may be related to decreasing organism load in the effluent.

The strengths of this study include its size, with 221 CLABSIs and $>140,000$ central-line days, and design. The use of 2 hospitals that switched NCs at a predetermined time point helped compensate for changes in practices over time that have confounded many quasi-experimental studies. A randomized controlled trial of this size involving all patients at 2 large hospitals is not practical. In addition, the NCs were mechanically identical, so no changes in nursing workflow related to clamping or flushing the device impacted study outcomes. The majority of CLABSIs now occur outside ICUs. ${ }^{1,31,34}$ We were able to include all central lines inside and outside of ICUs, and we used a previously validated methodology to calculate the number of central-line days outside the ICUs.

Failure to adequately clean hubs prior to accession and NC design flaws, such as crevices, likely contribute to CLABSIs via the intraluminal pathway. Although guidelines stress the importance of cleaning NCs, ${ }^{10}$ the optimal time for disinfecting the NC hub by scrubbing prior to accession is an unresolved issue. ${ }^{35}$ Technology alone should not be used to compensate for suboptimal NC cleaning practices and should be used synergistically with efforts to promote device cleaning. We received anecdotal reports of decreased compliance with hub cleaning prior to accessing the silver-coated NC, 


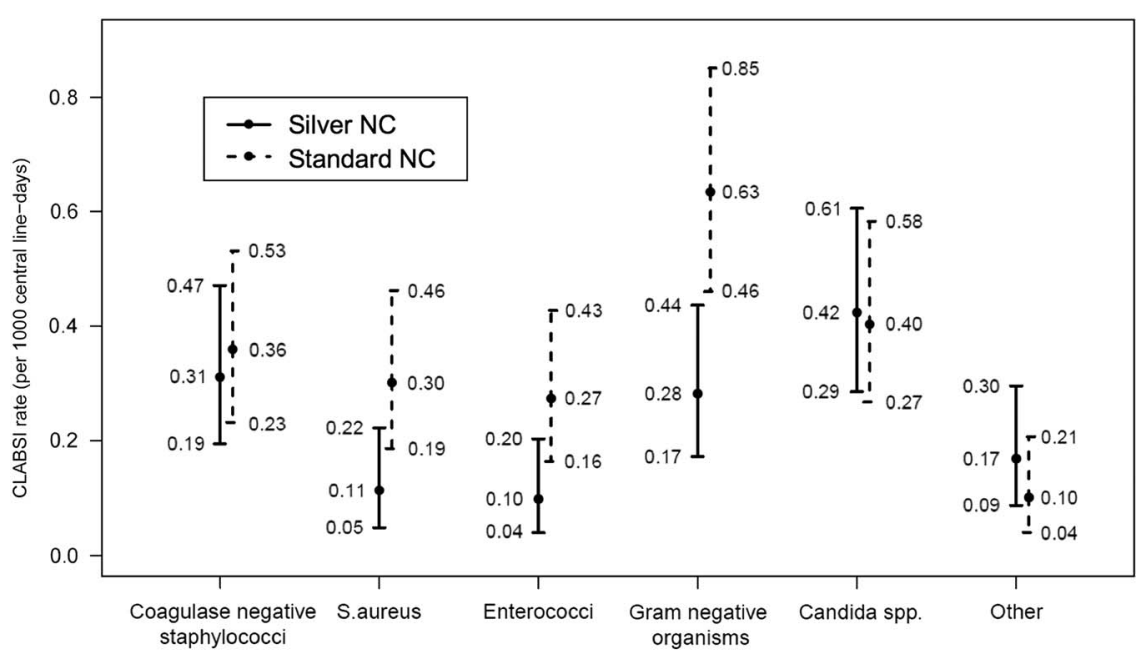

FIGURE 1. Comparison of the distribution of organism-specific central line-associated bloodstream infection (CLABSI) by needleless connector (NC). Vertical bars indicate the $95 \%$ confidence intervals.

rationalized by its potential protective effect. Thus, there may be unintended consequences of using antimicrobial devices, underscoring the need to maintain vigilance regarding clinical practices. We did not measure NC cleaning in our study, but decreased cleaning rigor of the silver-coated NC could lead to underestimating its effect on CLABSI rates.

We observed a significant reduction in CLABSI rates for S. aureus, enterococci, and Gram-negative bacilli using the silver-coated NC, but not for coagulase-negative staphylococci and yeast. We speculate that the lack of reduction in coagulasenegative staphylococcal CLABSIs was related to many of these infections being extraluminal in origin and likely insertionrelated events not amenable to prevention by an antimicrobial NC. However, measuring the time of line insertion to infection stratified by organism type did not reveal a clustering of coagulase-negative staphylococcal infections close to the date of insertion. Thus, the reasons that the silver-coated NC device had no impact on coagulase-negative staphylococcal and Candida infections are unclear, although the lack of specificity of the NHSN CLABSI definition could have contributed to our findings. Alternatively, intrinsic properties of Candida spp. and coagulase-negative staphylococci or their extracellular polymers may affect their susceptibility to the silver.

The analysis of time from central-line insertion to CLABSI stratified by organism type showed no clustering of infection caused by common skin organisms, such as coagulase-negative staphylococci, in the first 7-10 days after line insertion. Prior efforts to optimize central-line insertion practices may contribute to this finding although NCs may become contaminated by cutaneous organisms, leading to late-onset CLABSIs. ${ }^{7}$

We are aware of several limitations in this study. The setting, 2 university hospitals, may not be generalizable. Hospitals with lower intensity of care and greater reduction of CLABSIs through other interventions would have different inputs in a cost-effectiveness analysis. However, the size and the inclusion of all admitted adult patients make this a more comprehensive study than those limited to ICUs. While no major changes in infection prevention activities or introduction of new equipment were implemented during the course of the study, unitlevel process-improvement activities to prevent CLABSIs were ongoing at both hospitals. The magnitude of such improvements are unlikely to have affected the study end point, and the device switch should mitigate impact of general improvement efforts over time. The surveillance CLABSI definition, which prioritizes sensitivity over specificity, could have led to an underestimation of the reduction of true central-line-related infections due to the silver-coated NCs. In particular, the surveillance definition of CLABSI, including all bloodstream infections without a known primary source occurring in patients with central lines, lacks specificity, particularly in patients with chemotherapy-induced neutropenia who are predisposed to translocation of intestinal organisms. ${ }^{24}$ This study was conducted before the CDC developed a modification of the surveillance CLABSI definition to account for patients with mucosal barrier injury and neutropenia. ${ }^{27} \mathrm{We}$ compensated for the lack of specificity of the contemporary surveillance CLABSI definition in patients with neutropenia by excluding those patients from the numerator in a sensitivity analysis. In this analysis, we likely excluded some true CLABSIs and were unable to remove central-line days during neutropenia, which could also lead to an underestimation of the effect of silver-coated NCs.

An important finding of our sensitivity analysis is the relative change in CLABSI rate in hospital $\mathrm{B}$ compared with hospital A when patients with neutropenia were excluded. This finding underscores the pitfalls of using the current surveillance definition for interinstitutional benchmarking. The recent CDC effort to modify the CLABSI definition is an important step that, in the future, may allow for more meaningful comparisons between hospitals providing services 
to patients with neutropenia following chemotherapy and those with smaller immunocompromised populations.

The relative contribution of the intraluminal compared with the extraluminal pathway to CLABSI remains unclear. ${ }^{36}$ This study adds to the understanding of the pathogenesis of CLABSIs and demonstrates that at least 30\% of CLABSIs are acquired by hub contamination and are preventable. The decreased CLABSI rate in patients in whom silver-coated NCs were used versus those in whom standard NCs were used adds to the growing evidence that CLABSI prevention programs should focus not only on insertion practices but also on practices and devices that prevent catheter hub contamination and intraluminal infection, such as hand hygiene and scrubbing the hub.

\section{ACKNOWLEDGMENTS}

We wish to thank the staff and leadership in nursing and materials management at Emory University Hospital Midtown and Emory University Hospital for their valued participation and support.

Financial support: This work was supported by an investigator-initiated grant from Baxter Healthcare to JTJ, SCT, and JPS. The study sponsor was not involved in the design and conduct of the study; collection, management, analysis, and interpretation of the data; and preparation, review, or approval of the manuscript.

Potential conflicts of interest: MDR, XL, KAE, WLA, GG, KG, CH, and CR report no conflicts of interests relevant to this article.

Address correspondence to Jesse T. Jacob, MD, Orr Building \#1020, 550 Peachtree Street NE, Atlanta, GA 30308. (jtjacob@emory.edu).

\section{REFERENCES}

1. Centers for Disease Control and Prevention. Vital signs: central line-associated blood stream infections-United States, 2001, 2008, and 2009. Morb Mortal Wkly Rep 2011;60:243-248.

2. Mermel LA, McCormick RD, Springman SR, Maki DG. The pathogenesis and epidemiology of catheter-related infection with pulmonary artery Swan-Ganz catheters: a prospective study utilizing molecular subtyping. Am J Med 1991;91:197S-205S.

3. Linares J, Sitges-Serra A, Garau J, Perez JL, Martin R. Pathogenesis of catheter sepsis: a prospective study with quantitative and semiquantitative cultures of catheter hub and segments. J Clin Microbiol 1985;21:357-360.

4. Almirall J, Gonzalez J, Rello J, et al. Infection of hemodialysis catheters: incidence and mechanisms. Am J Nephrol 1989;9:454-459.

5. Cheesbrough JS, Finch RG, Burden RP. A prospective study of the mechanisms of infection associated with hemodialysis catheters. J Infect Dis 1986;154:579-589.

6. Anaissie E, Samonis G, Kontoyiannis D, et al. Role of catheter colonization and infrequent hematogenous seeding in catheterrelated infections. Eur J Clin Microbiol 1995;14:134-137.

7. Safdar N, Maki DG. The pathogenesis of catheter-related bloodstream infection with noncuffed short-term central venous catheters. Intensive Care Med 2004;30:62-67.

8. Weightman NC, Simpson EM, Speller DC, Mott MG, Oakhill A. Bacteraemia related to indwelling central venous catheters: prevention, diagnosis and treatment. Eur J Clin Microbiol 1988;7:125-129.
9. Pronovost P, Needham D, Berenholtz S, et al. An intervention to decrease catheter-related bloodstream infections in the ICU. New Eng J Med 2006;355:2725-2732.

10. O'Grady NP, Alexander M, Burns LA, et al. Guidelines for the prevention of intravascular catheter-related infections. Clin Infect Dis 2011;52:e162-e193.

11. Maragakis LL, Bradley KL, Song X, et al. Increased catheterrelated bloodstream infection rates after the introduction of a new mechanical valve intravenous access port. Infect Control Hosp Epidemiol 2006;27:67-70.

12. Field $\mathrm{K}, \mathrm{McF}$ arlane $\mathrm{C}$, Cheng $\mathrm{AC}$, et al. Incidence of catheterrelated bloodstream infection among patients with a needleless, mechanical valve-based intravenous connector in an Australian hematology-oncology unit. Infect Control Hosp Epidemiol 2007;28:610-613.

13. Salgado CD, Chinnes L, Paczesny TH, Cantey JR. Increased rate of catheter-related bloodstream infection associated with use of a needleless mechanical valve device at a long-term acute care hospital. Infect Control Hosp Epidemiol 2007;28:684-688.

14. Rupp ME, Sholtz LA, Jourdan DR, et al. Outbreak of bloodstream infection temporally associated with the use of an intravascular needleless valve. Clin Infect Dis 2007;44:1408-1414.

15. Liau SY, Read DC, Pugh WJ, Furr JR, Russell AD. Interaction of silver nitrate with readily identifiable groups: relationship to the antibacterial action of silver ions. Letters Appl Microbiol 1997;25:279-283.

16. Maillard JY, Hartemann P. Silver as an antimicrobial: facts and gaps in knowledge. Critical Rev Microbiol 2013;39:373-383.

17. Saint S, Elmore JG, Sullivan SD, Emerson SS, Koepsell TD. The efficacy of silver alloy-coated urinary catheters in preventing urinary tract infection: a meta-analysis. Am J Med. 1998;105: 236-241.

18. Casey AL, Mermel LA, Nightingale P, Elliott TSJ. Antimicrobial central venous catheters in adults: a systematic review and metaanalysis. Lancet Infect Dis 2008;8:763-776.

19. Maki DG. In vitro studies of a novel antimicrobial luer-activated needleless connector for prevention of catheter-related bloodstream infection. Clin Infect Dis 2010;50:1580-1587.

20. Edmiston CE Jr, Markina V. Reducing the risk of infection in vascular access patients: an in vitro evaluation of an antimicrobial silver nanotechnology luer activated device. Am J Infect Control 2010;38:421-423.

21. National Healthcare Safety Network (NHSN). Central lineassociated BSI (CLABSI) event. http://www.cdc.gov/nhsn/TOC_ PSCManual.html. Published in 2011. Accessed July 11, 2011.

22. Chernetsky Tejedor S, Garrett G, Jacob JT, et al. Electronic documentation of central venous catheter-days: validation is essential. Infect Control Hosp Epidemiol 2013;34:900-907.

23. Fraser TG, Gordon SM. CLABSI rates in immunocompromised patients: a valuable patient centered outcome? Clin Infect Dis 2011;52:1446-1450.

24. Sexton DJ, Chen LF, Anderson DJ. Current definitions of central line-associated bloodstream infection: is the emperor wearing clothes? Infect Control Hosp Epidemiol 2010;31:1286-1289.

25. Steinberg JP, Robichaux C, Tejedor SC, Reyes MD, Jacob JT. Distribution of pathogens in central line-associated bloodstream infections among patients with and without neutropenia following chemotherapy: evidence for a proposed modification to the current surveillance definition. Infect Control Hosp Epidemiol 2013;34:171-175. 
26. Worth LJ, Slavin MA, Brown GV, Black J. Catheter-related bloodstream infections in hematology: time for standardized surveillance? Cancer 2007;109:1215-1226.

27. See I, Iwamoto $M$, Allen-Bridson K, Horan T, Magill SS, Thompson ND. Mucosal barrier injury laboratory-confirmed bloodstream infection: results from a field test of a new National Healthcare Safety Network definition. Infect Control Hosp Epidemiol 2013;34:769-776.

28. Bailar JC, Ederer F. Significance factors for the ratio of a Poisson variable to its expectation. Biometrics 1964;20:639-643.

29. Brownlee KA. Statistical theory and methodology in science and engineering. New York: Wiley; 1960.

30. Rosner B. Fundamentals of biostatistics, 5th ed. Pacific Grove, CA: Duxbury, 1999.

31. Marschall J, Leone C, Jones M, Nihill D, Fraser VJ, Warren DK. Catheter-associated bloodstream infections in general medical patients outside the intensive care unit: a surveillance study. Infect Control Hosp Epidemiol 2007;28:905-909.
32. Casey AL, Karpanen TJ, Nightingale P, Cook M, Elliott TSJ. Microbiological comparison of a silver-coated and a non-coated needleless intravascular connector in clinical use. J Hosp Infect 2012;80:299-303.

33. Perez E, Williams M, Jacob JT, et al. Microbial biofilms on needleless connectors for central venous catheters: comparison of standard and silver-coated devices collected from patients in an acute care hospital. J Clin Microbiol 2014;52:823-831.

34. Climo M, Diekema D, Warren DK, et al. Prevalence of the use of central venous access devices within and outside of the intensive care unit: results of a survey among hospitals in the prevention epicenter program of the Centers for Disease Control and Prevention. Infect Control Hosp Epidemiol 2003;24:942-945.

35. Simmons S, Bryson C, Porter S. "Scrub the hub": cleaning duration and reduction in bacterial load on central venous catheters. Critical Care Nurs Q 2011;34:31-35.

36. Mermel LA. What is the predominant source of intravascular catheter infections? Clin Infect Dis 2011;52:211-212. 